\title{
Initiation of Coronal Mass Ejections by Sunspot Rotation
}

\author{
G. Valori ${ }^{1}$, T. Török ${ }^{2}$, M. Temmer ${ }^{3,4}$, A. M. Veronig ${ }^{3}$, \\ L. van Driel-Gesztelyi ${ }^{1,5,6}$, and B. Vršnak ${ }^{7}$ \\ ${ }^{1}$ LESIA, Observatoire de Paris, CNRS, UPMC, Université Paris Diderot, 5 place Jules \\ Janssen, 92190 Meudon, France; email: gherardo.valori@obspm.fr \\ ${ }^{2}$ Predictive Science Inc., 9990 Mesa Rim Rd., Suite 170, San Diego, CA 92121, USA; email: \\ tibor@predsci.com \\ ${ }^{3}$ IGAM/Kanzelhöhe Observatory, Institute of Physics, Universität Graz, Universitätsplatz 5, \\ A-8010 Graz, Austria \\ ${ }^{4}$ Space Research Institute, Austrian Academy of Sciences, Schmiedlstrasse 6, A-8042 Graz, \\ Austria \\ ${ }^{5}$ University College London, Mullard Space Science Laboratory, Holmbury St. Mary, Dorking, \\ Surrey RH5 6NT, UK \\ ${ }^{6}$ Konkoly Observatory, Hungarian Academy of Sciences, Budapest, Hungary \\ ${ }^{7}$ Hvar Observatory, Faculty of Geodesy, University of Zagreb, Kačićeva 26, HR-10000 Zagreb, \\ Croatia
}

\begin{abstract}
We report observations of a filament eruption, two-ribbon flare, and coronal mass ejection (CME) that occurred in Active Region NOAA 10898 on 6 July 2006. The filament was located South of a strong sunspot that dominated the region. In the evolution leading up to the eruption, and for some time after it, a counter-clockwise rotation of the sunspot of about 30 degrees was observed. We suggest that the rotation triggered the eruption by progressively expanding the magnetic field above the filament. To test this scenario, we study the effect of twisting the initially potential field overlying a pre-existing flux rope, using three-dimensional zero- $\beta$ MHD simulations. We consider a magnetic configuration whose photospheric flux distribution and coronal structure is guided by the observations and a potential field extrapolation. We find that the twisting leads to the expansion of the overlying field. As a consequence of the progressively reduced magnetic tension, the flux rope quasi-statically adapts to the changed environmental field, rising slowly. Once the tension is sufficiently reduced, a distinct second phase of evolution occurs where the flux rope enters an unstable regime characterized by a strong acceleration. Our simulation thus suggests a new mechanism for the triggering of eruptions in the vicinity of rotating sunspots.
\end{abstract}

Keywords. MHD, instabilities, Sun: activity, magnetic fields, sunspots, coronal mass ejections (CMEs), filaments, methods: numerical, data analysis

\section{Introduction}

The eruption on 6 July 2006 in active region NOAA 10898 was a two-ribbon flare accompanied by a filament eruption and a halo $\mathrm{CME}$, the latter being most prominent in the southwest quadrant and reaching a linear plane-of-sky velocity of $\approx 900 \mathrm{~km} \mathrm{~s}^{-1}$ (Temmer et al. 2008). The event was associated with an EIT wave, a type II burst, and very distinct coronal dimming regions. The flare was of class $\mathrm{M} 2.5 / 2 \mathrm{~N}$, located at the heliographic position $\mathrm{S} 9^{\circ}, \mathrm{W} 34^{\circ}$. It was observed in soft X-rays (SXR) by GOES (peak time at $\approx 08: 37 \mathrm{UT}$ ) as well as in hard-X rays (HXR) with RHESSI, with the two highest peaks of nonthermal HXR emission occurring during 08:20-08:24 UT.

The morphology and evolution of the bipolar active region in the days preceding the eruption were studied using photospheric line-of-sight magnetograms obtained by the 

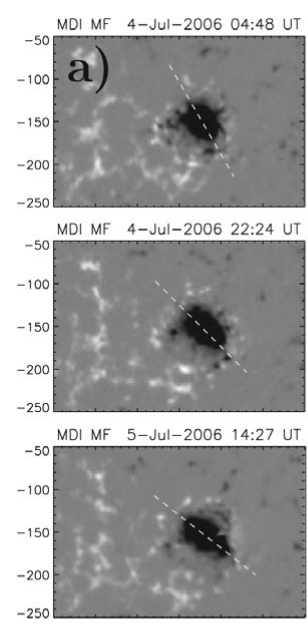

MDI MF 6-Jul-2006 09:35 UT

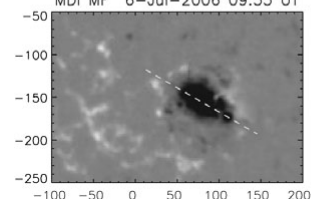

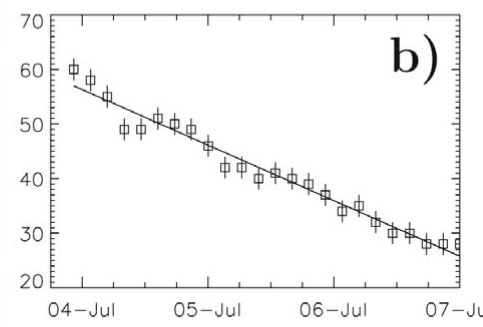

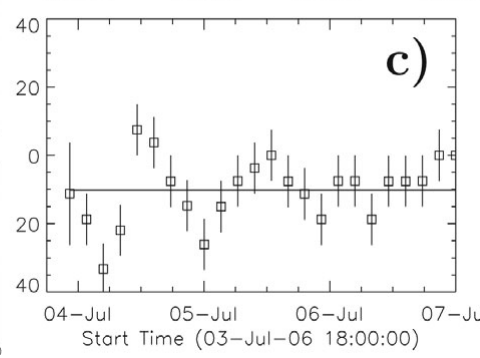

Figure 1. (a) Representative MDI longitudinal magnetic-field maps of the sunspot evolution during 4-6 July 2006: The dashed yellow line outlines the major axis of the sunspot that was used to measure the sunspot rotation. The images are all differentially rotated to the first image of the series, when the sunspot was closer to disk centre. (b) Sunspot rotation determined from the MDI magnetic-field maps over the period 3 July 2006, 22:00 UT, to 7 July 2006, 8:00 UT, showing the orientation of the sunspot's major axis, measured clockwise from solar East. (c) Sunspot rotation rate in degrees per day, determined as the temporal derivative of the rotation measurements.

MDI instrument (Scherrer et al. 1995). The region consisted of a compact negative polarity (the sunspot) surrounded by a dispersed positive polarity, most of which was extending eastwards (Fig. 1a). The two polarities were surrounded by a large, "inverse C-shaped" area of dispersed negative flux to the west of the region (Fig. 2a). The magnetic-flux measurements indicate a mere $5 \%$ negative surplus flux in this major bipolar active region of $2.1 \times 10^{22} \mathrm{Mx}$ total flux and maximum-field strengths (negative:positive) in a roughly 10:1 ratio. The sequence in Fig. 1a shows that the sunspot is rotating counter-clockwise during the considered period. The total rotation observed over the three days preceding the eruption is about $30^{\circ}$, with sunspot's rotation rate of about $10^{\circ}$ day $^{-1}$ (Fig. $1 \mathrm{~b}, \mathrm{c}$ ).

The flare and the filament eruption were observed in full-disk $\mathrm{H} \alpha$ filtergrams by the Kanzelhöhe Observatory and, over a smaller field-of-view around the active region, by the Hvar Observatory. These observations reveal that the filament consisted of a double structure before and during the eruption. Significant rising motions of the filament could be seen from about 08:23 UT. The $\mathrm{H} \alpha$ flare started by the appearance of very weak double-footpoint brightening at 08:15 UT. We also estimated the kinematics of the filament and the CME front from a time sequence of running-difference images obtained from TRACE, EIT, and LASCO C2/C3 observations. We obtain that the coronal loops overlying the filament started their slow rising phase at 08:15 UT, i.e., about five-ten minutes before the filament. Similarly, the CME front reached its final, almost constant velocity a few minutes before the filament. More details about the methods employed to obtain the above results can be found in Török et al. (2013), hereafter Paper I. We refer to that article and to the references therein for further details on the eruption.

Guo et al. (2010) suggested that the eruption was triggered by recurrent chromospheric mass injection in the form of surges or jets into the filament channel. Here we propose a different mechanism: Assuming that the filament was suspended in the corona by a magnetic flux rope, we suggest that the continuous rotation of the sunspot led to a slow expansion of the arcade-like magnetic field overlying the filament, i.e., to a continuous weakening of its stabilizing tension, until a critical point was reached at which equilibrium 

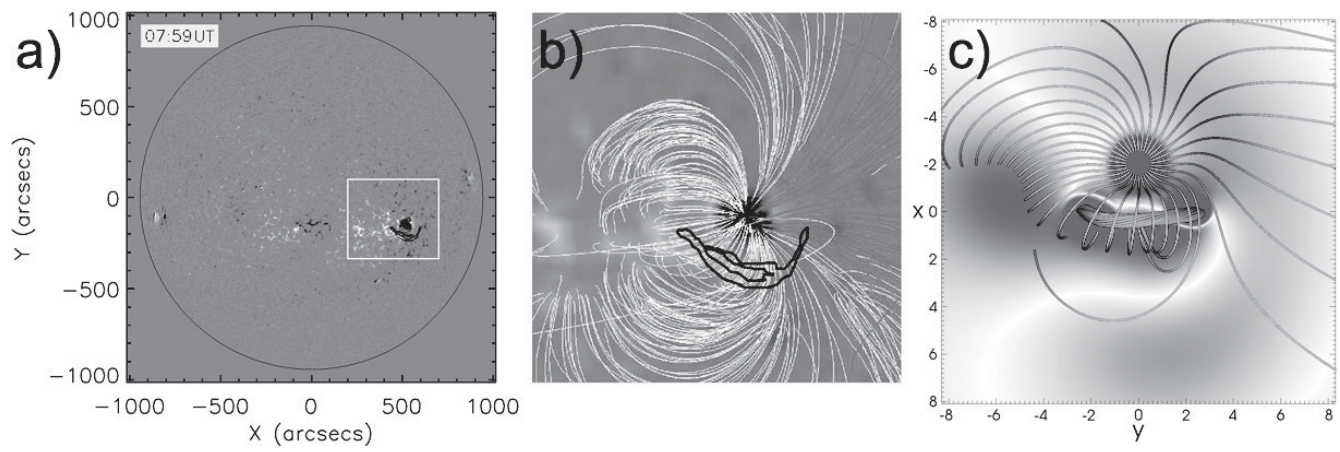

Figure 2. (a) Full-disk MDI magnetogram on 6 July 2006, 07:59 UT. AR10898 is marked by the white box. (b) PFSS magnetic field lines in the AR calculated for 6 July 2006, 06:04 UT, overlaid on a synoptic MDI magnetogram. Pink (white) field lines depict open (closed) fields. The outer contours of the filament, based on $\mathrm{H} \alpha$ data taken at 07:59 UT on 6 July 2006, are outlined with black lines. (c) Magnetic configuration used in the simulation, after the initial relaxation of the system, showing the core of the TD flux rope (orange field lines) and the ambient potential field (green field lines). $B_{z}(z=0)$ is shown, with red (blue) corresponding to positive (negative) values, and is saturated at $4 \% \max \left(B_{z}\right)$ to depict weaker flux distributions.

could not be maintained and the flux rope erupted. We note that we do not claim that the eruption was triggered exclusively by this mechanism. Filaments are often observed to spiral into the periphery of sunspots (see,e.g., Green et al. 2007), and also in our case an inspection of the TRACE and $\mathrm{H} \alpha$ images during the early phase of the eruption suggests a possible magnetic connection between the western extension of the filament-carrying core field and the sunspot area. Thus, the sunspot rotation may have added stress to this field, thereby possibly contributing to drive it towards eruption. On the other hand, for an injection of twist to occur, the core field must be rooted in the centre of the sunspot, not just in its periphery, which is difficult to establish from observations. It appears reasonable to assume that a clear connection between core field and sunspot centre is not always present, and that the stressing of the overlying ambient field by sunspot rotation may be more relevant for the destabilization of the system in such cases. In order to test this scenario, we perform a three-dimensional (3D) MHD simulation in which we twist the stabilizing potential field overlying a stable coronal flux rope. Differently from previous works (e.g., Amari et al. 1996), the photospheric vortex motions we use do not directly affect the flux rope, but solely the field surrounding it.

\section{Numerical simulation setup}

As in previously published simulations (e.g., Török, Kliem, and Titov 2004; Kliem, Titov, and Török 2004), we integrate the $\beta=0$ compressible ideal MHD equations, ignoring the effects of thermal pressure and gravity, and we employ the coronal flux rope model of Titov and Démoulin (1999), hereafter TD, to construct the initial magnetic field. The main ingredient of the TD model is a current ring of major radius $[R]$ and minor radius $[a]$ that is placed such that its symmetry axis is located at a depth $[d]$ below a photospheric plane. The outwardly directed Lorentz self-force (or "hoop force") of the ring is balanced by a potential field created by a pair of sub-photospheric point sources $\pm q$ that are placed at the symmetry axis, at distances $\pm L$ from the ring centre. The resulting coronal field consists of an arched and line-tied flux rope embedded in an arcade-like potential field. 
We normalize lengths by $l=R-d$ and use a Cartesian grid discretizing the volume $[-40,40] \times[-30,30] \times[0,60]$, resolved by $307 \times 257 \times 156$ points. The grid is nonuniform in all directions, with an almost uniform resolution $\Delta=0.05$ in the area mimicking the active region. The top and lateral boundaries are closed, which is justified given the large size of the simulation box. Below the photospheric plane, the tangential components of the magnetic field $\left[B_{x, y}\right]$ are extrapolated from the integration domain, and the normal component $\left[B_{z}\right]$ is set such that $\nabla \cdot \mathbf{B}=\mathbf{0}$ in $z=0$ at all times. The vertical velocities are zero there at all times, and the mass density is fixed at its initial values.

Fig. 2 b shows a coronal potential-field source-surface model (Schatten, Wilcox, and Ness 1969, PFSS), obtained from a synoptic MDI magnetogram for Carrington Rotation 2045. It can be seen that the field lines rooted in the main polarity (the sunspot) form a fan-like structure, which partly overlies the pre-eruption filament. In order to build an initial magnetic configuration that resembles this coronal field and the underlying highly asymmetric magnetic flux distribution (Sect. 1), we modify the standard TD model by replacing the pair of sub-photospheric point charges by an ensemble of ten sub-photospheric sources. These are adjusted in order to mimic: the approximate flux balance between the concentrated leading negative polarity and the dispersed following positive polarity; the ratio of approximately 10:1 between the peak field strengths in the leading polarity and the following polarity; the size ratio between these polarities; the presence of an "inverse C-shaped" area of dispersed negative flux to the West of the leading polarity; the fan-like shape of the coronal field rooted in the leading polarity. Since the model is still relatively idealized, all these features can be matched only approximately. We then add a TD flux rope, setting $R=2.75, a=0.8$, and $d=1.75$. The position of the rope within the ambient field is guided by the observed location of the filament (Fig. 2b), and its magnetic field strength is chosen such that it is in approximate equilibrium with the ambient potential field. We use an initial density distribution $\rho_{0}(\mathbf{x})=\left|\mathbf{B}_{\mathbf{0}}(\mathbf{x})\right|^{2}$, corresponding to a uniform initial Alfvén velocity. In order to obtain a numerical equilibrium as a starting point, we first perform a numerical relaxation for $75 \tau_{a}$, after which the time is reset to zero.

In order to mimic the observed sunspot rotation, we then twist the main negative flux concentration by imposing tangential velocities at the bottom boundary. They produce a horizontal counterclockwise rotation, chosen such that the velocity vectors always point along the contours of $B_{z}(x, y, 0, t=0)$, which assures that the distribution of $B_{z}(x, y, 0, t)$ is conserved to a very good approximation. The velocities are zero at the polarity centre, located at $(x, y, z)=(-2,0,0)$, and decrease towards its edge from their maximum value, equal to 0.005 times the initial Alfvén velocity $\left[v_{a 0}\right]$. The equations and parameters used to compute the tangential velocities at each time are given in Paper I. The twist injected by such motions is nearly uniform close to the polarity centre and decreases monotonically towards its edge, such that it does not directly affect the flux rope field.

\section{Results}

The magnetic configuration resulting after the initial numerical relaxation is shown in Fig. 2c and in Fig. 4a. The fan-structure inferred from the PFSS extrapolation is qualitatively well reproduced. The TD flux rope is stabilized by flux rooted towards the southern edge of the main polarity, and the rope is inclined with respect to the vertical, which is due to the asymmetry of the potential field surrounding it.

Figure 4a shows that electric currents are present in the ambient field volume. The strongest current concentrations are located in the front of the flux rope and exhibit an $\mathrm{X}$-shaped pattern in the vertical cut shown. This pattern outlines the locations of quasiseparatrix layers (QSL, see, e.g., Démoulin et al. 1996) that separate different connectivity 


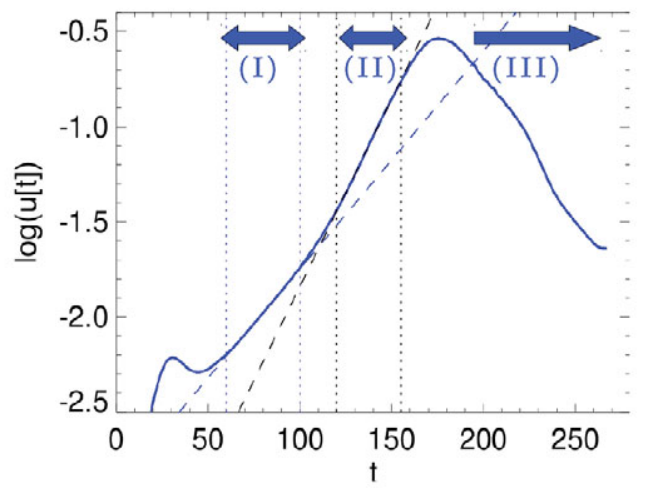

Figure 3. Logarithmic presentation of the velocity of the axis apex of the TD flux rope during the twisting phase, as a function of time. The dashed lines show linear fits, obtained within the time periods marked by the vertical dotted lines of the same color. Thick arrows mark different evolution phases described in the text.

domains. The QSLs are present in the configuration from the very beginning and arise from the complexity of the potential field. Their presence is evident also in the left panel of Fig. 4a: the green field lines show strong connectivity gradients in the northern part of the main polarity and in the vicinity of the western flux rope footpoint. It has been demonstrated that current concentrations form preferably at the locations of QSLs as a system containing such structures is dynamically perturbed (see, e.g., Aulanier, Pariat, and Démoulin 2005). In our case the perturbation results from the - relatively modest dynamics during the initial relaxation of the system.

After the relaxation, at $t=0$, we start twisting the main negative polarity, and we quantify the evolution of the TD flux rope by monitoring the velocity at the axis apex of the rope (Fig. 3). Due to the pronounced fan-structure of the field rooted in the main polarity, the photospheric twisting does not lead to the formation of a single twisted flux tube that rises exactly in vertical direction above the TD rope, as it was the case earlier studies (Amari et al. 1996; Török and Kliem 2003; Aulanier, Démoulin, and Grappin 2005). Rather, the twisting leads to a slow, global expansion of the fan-shaped field lines, as shown in Fig. 4. Since we are mainly interested in the destabilization of the flux rope, we did not study the detailed evolution of the large-scale field. We expect it to be very similar to the one described in Santos, Büchner, and Otto (2011), since the active region those authors simulated was also dominated by one main polarity, and the field rooted therein had a very similar fan-shaped structure (cf. our Fig. 4 with their Fig.1).

Important for our purpose is the evolution of the arcade-like part of the initial potential field that directly overlies the TD flux rope. Those field lines are directly affected only by a fraction of the boundary flows and therefore get merely sheared (rather than twisted), which still leads to their slow expansion. As a result, the TD rope starts to rise, adapting to the successively decreasing magnetic tension of the overlying field (phase I in Fig. 3). This initial phase of the evolution is depicted in Fig. 4b. Note that some of the flux at the front of the expanding arcade reconnects at the QSL current layer, which can be expected to aid the arcade expansion to some degree. As can be seen in Fig. 3, the TD rope rises, after some initial adjustment, exponentially during this slow initial phase.

As the twisting continues, a transition to a rapid acceleration takes place after $t \approx$ $100 \tau_{a}$, when the rise curve leaves the quasi-static regime. After the transition phase, the TD rope again rises exponentially, but now with a significantly larger growth rate than during the slow rise phase (phase II in Fig. 3). Such a slow (quasi-static) rise phase, followed by a rapid acceleration, is a well-observed property of many filament eruptions in the early evolution of CMEs (see, e.g., Schrijver et al. 2008, and references therein), and is also seen for the event studied here (see also Paper I). The evolution of the TD rope after $t \approx 100 \tau_{a}$ can be associated with the development of the torus instability 


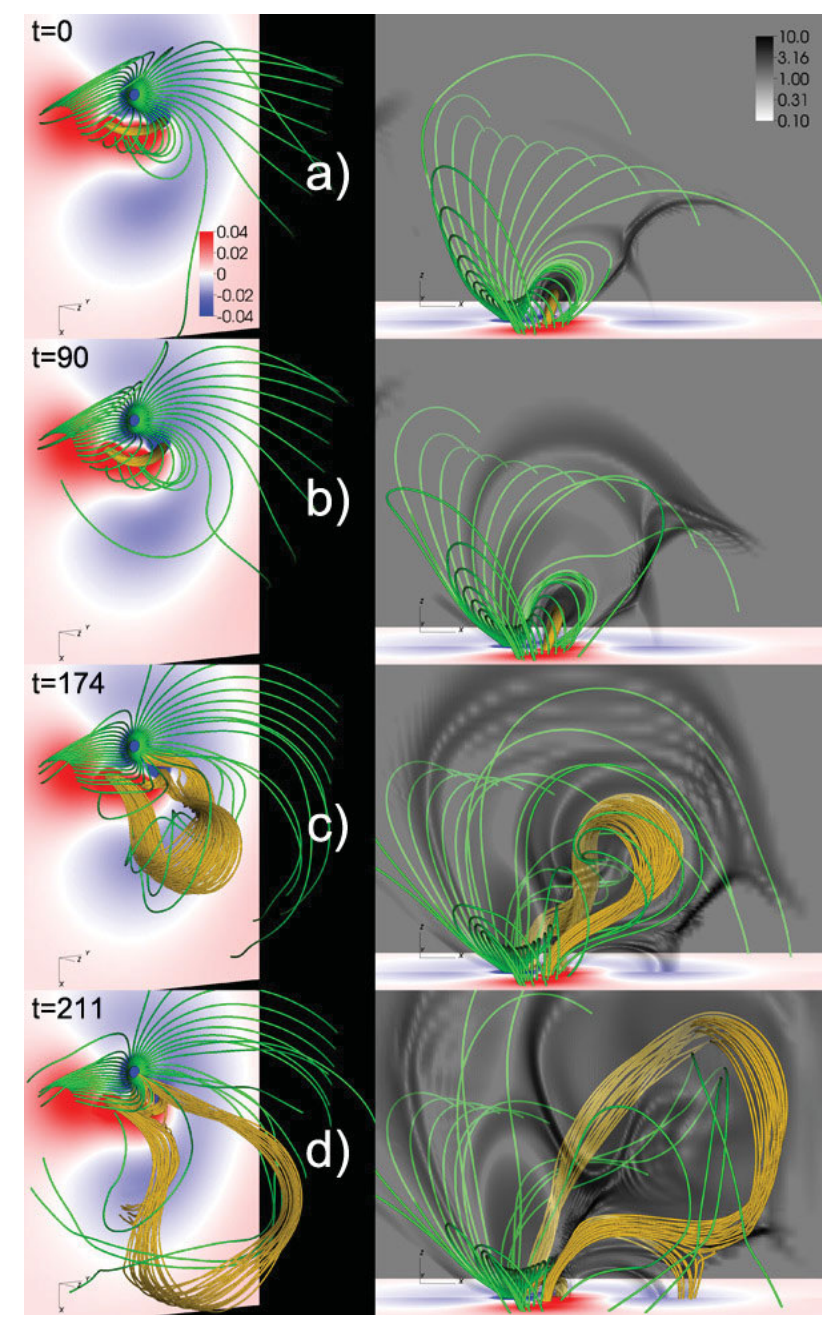

Figure 4. Magnetic configuration after initial relaxation (a), during slow rise phase (b), at time of peak flux rope velocity (c), and during flux rope deceleration (d). The flux rope core is depicted by orange field lines; ambient field lines are green. $B_{z}(z=0)$ is shown, with red (blue) corresponding to positive (negative) values. Left panels use a view similar to the observations (see paper I); right panels show a side view. The transparent grey-scales show a logarithmic distribution of $|\mathbf{j}| /|\mathbf{B}|$ in the plane $x=0$, outlining the locations of strongest currents. The sub-volume $[-10,16] \times[-11,11] \times[0,18]$ is used for all panels.

(Kliem and Török 2006; Démoulin and Aulanier 2010), as has been shown under similar conditions in various simulations of erupting flux ropes (Török and Kliem 2007; Fan and Gibson 2007; Schrijver et al. 2008; Aulanier et al. 2010; Török et al. 2011). The right panels in Fig. 4 show that the trajectory of the flux rope is far from being vertical. Such lateral eruptions have been reported frequently in both observations and simulations (see, e.g., Williams et al. 2005; Panasenco et al. 2011; Yang et al. 2012), and are usually attributed to an asymmetric structure of the field overlying the erupting core flux. We believe that this causes the lateral rise also in our case.

As the eruption continues, the trajectory of the flux rope becomes increasingly horizontal, resembling the so-called "roll effect" (Panasenco et al. 2011) and indicating that the rope cannot overcome the tension of the large-scale overlying field. Moreover, as a 
consequence of its increasing expansion, the flux rope strongly pushes against the QSL current layer, which results in reconnection between the front of the rope and the ambient field. Eventually, the rope splits into two parts, similar to what has been found in simulations of confined eruptions (Amari and Luciani 1999; Török and Kliem 2005). These two effects slow down the rise of the rope after $t \approx 175 \tau_{a}$ and inhibit its full eruption, i.e., the development of a CME in our simulation (phase III in Fig. 3).

Since QSLs can affect the evolution of an eruption, but are not expected to play a significant role for its initiation, we did not investigate in detail whether or not QSLs were present in the pre-eruption configuration of the 6 July 2006 event. The PFSS extrapolation indicates the presence of a QSL to the North and the West of the main polarity (see the field-line connectivities in Fig. 2b), but less clearly so to its South. Since we merely aim to model the initiation of the eruption rather than its full evolution into a CME, we refrained from further improving our model to obtain a configuration without a strong QSL in front of the flux rope.

\section{Summary and Conclusions}

We presented a 3D MHD simulation that was designed to test a possible scenario for the initiation and early evolution of the filament eruption and CME that occurred on 6 July 2006 in active region NOAA 10898. Our conjecture was that the slow rotation of the sunspot that dominated the active region progressively reduced the tension of the magnetic field overlying the pre-eruption filament, until the latter could not be stabilized anymore and erupted, resulting in the CME. Using the TD coronal flux rope model as a starting point, we constructed an initial magnetic field that resembles the photospheric flux distribution and coronal magnetic field structure of the active region around the time of the event. In particular, the highly asymmetric flux density and the resulting overall fan-shape of the coronal magnetic field are well captured by the model, while the approximative flux balance of the region is kept. We then mimicked the observed sunspot rotation by imposing photospheric vortex flows localized at the main magnetic polarity of the model. The flows were chosen such that they do not directly affect the flux rope.

As a result of this twisting, the field lines overlying the flux rope start to expand and the rope undergoes a quasi-static adaptation to the changing surrounding field in the simulation, which manifests in a slow rise phase. As the weakening of the overlying field reaches an appropriate level, the torus instability sets in and rapidly accelerates the rope upwards, leading to a second, fast rise phase and eruption. The asymmetry of the ambient field leads to a markedly lateral eruption. This evolution in two phases resembles the often observed slow rise phase and subsequent strong acceleration of filaments in the course of their eruption. However, the presence of a QSL-related current layer in the front of the erupting flux rope in the simulation results in magnetic reconnection which eventually splits the rope before it can evolve into a CME, in contrast to the observations. Although we are not able to follow the expansion of the flux rope beyond this phase, the simulation successfully models the early phases of the eruption (the slow rise and the initial rapid acceleration of the flux rope) in a setting that is qualitatively similar to the observed magnetic configuration around the time of the eruption.

Our simulation thus demonstrates that the continuous expansion due to sunspot rotation of the magnetic field that stabilizes the current-carrying core flux, i.e., the progressive decrease of magnetic tension, can lead to filament eruptions and CMEs.

\section{Acknowledgements}

We acknowledge the use of data provided by the SOHO/MDI consortium. SOHO/EIT was funded by CNES, NASA, and the Belgian SPPS. The SOHO/LASCO data used here 
are produced by a consortium of the Naval Research Laboratory(USA), Max-PlanckInstitut für Aeronomie (Germany), Laboratoire d'Astrophysique de Marseille (France), and the University of Birmingham (UK). SOHO is a mission of international cooperation between ESA and NASA. The Transition Region and Coronal Explorer (TRACE) is a mission of the Stanford-Lockheed Institute for Space Research, and part of the NASA Small Explorer program. H $\alpha$ data were provided by the Kanzelhöhe Observatory, University of Graz, Austria, and by the Hvar Observatory, University of Zagreb, Croatia. The research leading to these results has received funding from the European Commission's Seventh Framework Programme (FP7/2007-2013) under the grant agreements $n^{\circ} 218816$ (SOTERIA project, www.soteria-space.eu) and $n^{\circ} 284461$ (eHEROES, http://soteriaspace.eu/eheroes/html). TT was partially supported by NASA's HTP, LWS, and SR\&T programs. LvDG acknowledges funding through the Hungarian Science Foundation grant OTKA K81421.

\section{References}

Amari, T. \& Luciani, J. F. 1999, Astrophys. J. Lett. 515, L81.

Amari, T., Luciani, J. F., Aly, J. J., \& Tagger, M. 1996, Astrophys. J. Lett. 466, L39.

Aulanier, G., Démoulin, P., \& Grappin, R. 2005, Astron. Astrophys. 430, 1067.

Aulanier, G., Pariat, E., \& Démoulin, P. 2005, Astron. Astrophys. 444, 961.

Aulanier, G., Török, T., Démoulin, P., \& DeLuca, E. E. 2010, Astrophys. J. 708, 314.

Démoulin, P. \& Aulanier, G. 2010, Astrophys. J. 718, 1388.

Démoulin, P., Henoux, J. C., Priest, E. R., \& Mandrini, C. H. 1996, Astron. Astrophys. 308, 643.

Fan, Y. \& Gibson, S. E. 2007, Astrophys. J. 668, 1232.

Gopalswamy, N., Yashiro, S., Kaiser, M. L., Howard, R. A., \& Bougeret, J. L. 2001, Astrophys. J. Lett. 548, L91.

Green, L. M., Kliem, B., Török, T., van Driel-Gesztelyi, L., \& Attrill, G. D. R. 2007, Solar Phys. 246,365 .

Guo, J., Liu, Y., Zhang, H., Deng, Y., Lin, J., \& Su, J. 2010, Astrophys. J. 711, 1057.

Kliem, B. \& Török, T. 2006, Phys. Rev. Lett. 96(25), 255002.

Kliem, B., Titov, V. S., \& Török, T. 2004, Astron. Astrophys. 413, L23.

Panasenco, O., Martin, S., Joshi, A. D., \& Srivastava, N. 2011, J. Atmos. Solar-Terr. Phys. 73, 1129.

Santos, J. C., Büchner, J., \& Otto, A. 2011, Astron. Astrophys. 535, A111.

Schatten, K. H., Wilcox, J. M., \& Ness, N. F. 1969, Solar Phys. 6, 442.

Scherrer, P. H., Bogart, R. S., Bush, R. I., Hoeksema, J. T., Kosovichev, A. G., Schou, J., Rosenberg, W., Springer, L., Tarbell, T. D., Title, A., Wolfson, C. J., \& Zayer, I., MDI Engineering Team 1995, Solar Phys. 162, 129.

Schrijver, C. J., Elmore, C., Kliem, B., Török, T., \& Title, A. M. 2008, Astrophys. J. 674, 586.

Temmer, M., Veronig, A. M., Vršnak, B., Rybák, J., Gömöry, P., Stoiser, S., \& Maričić, D. 2008, Astrophys. J. Lett. 673, L95.

Titov, V. S. \& Démoulin, P. 1999, Astron. Astrophys. 351, 707.

Török, T. \& Kliem, B. 2003, Astron. Astrophys. 406, 1043.

Török, T. \& Kliem, B. 2005, Astrophys. J. Lett. 630, L97.

Török, T. \& Kliem, B. 2007, Astronom. Nachr. 328, 743.

Török, T., Kliem, B., \& Titov, V. S. 2004, Astron. Astrophys. 413, L27.

Török, T., Panasenco, O., Titov, V. S., Mikić, Z., Reeves, K. K., Velli, M., Linker, J. A., \& De Toma, G. 2011, Astrophys. J. Lett. 739, L63.

Török, T., Temmer, M., Valori, G., Veronig, A. M., van Driel-Gesztelyi, L., \& Vršnak, B. 2013, Solar Phys. 286, 453. referred to as Paper I

Williams, D. R., Török, T., Démoulin, P., van Driel-Gesztelyi, L., \& Kliem, B. 2005, Astrophys. J. Lett. 628, L163.

Yang, J., Jiang, Y., Yang, B., Zheng, R., Yang, D., Hong, J., Li, H., \& Bi, Y. 2012, Solar Phys. 279, 115 . 